\title{
ORIGINAL ARTICLE \\ The influence of parental effects on transcriptomic landscape during early development in brook charr (Salvelinus fontinalis, Mitchill)
}

\author{
B Bougas ${ }^{1}$, C Audet ${ }^{2}$ and L Bernatchez ${ }^{1}$ \\ Parental effects represent an important source of variation in offspring phenotypes. Depending on the specific mechanisms \\ involved, parental effects may be caused to different degrees by either the maternal or the paternal parent, and these effects \\ may in turn act at different stages of development. To detect parental effects acting on gene transcription regulation and length \\ phenotype during ontogeny, the transcriptomic profiles of two reciprocal hybrids from Laval $\times$ Rupert and Laval $\times$ Domestic \\ populations of brook charr were compared at hatching, yolk sac resorption and 15 weeks after exogenous feeding. Using a \\ salmonid cDNA microarray, our results show that parental effects modulated gene expression among reciprocal hybrids only at \\ the yolk sac resorption stage. In addition, Laval $\times$ Domestic and Laval $\times$ Rupert reciprocal hybrids differed in the magnitude of \\ theses parental effects, with 199 and 630 differentially expressed transcripts, respectively. This corresponds to a maximum of \\ $18.5 \%$ of the analyzed transcripts. These transcripts are functionally related to cell cycle, nucleic acid metabolism and \\ intracellular protein traffic, which is consistent with observed differences associated with embryonic development and growth \\ differences in other fish species. Our results thus illustrate how parental effects on patterns of gene transcription seem \\ dependent on the genetic architecture of the parents. In addition, in absence of transcriptional differences, non-transcript \\ deposits in the yolk sac could contribute to the observed length differences among the reciprocal hybrids before yolk sac \\ resorption.
}

Heredity (2013) 110, 484-491; doi:10.1038/hdy.2012.113; published online 9 January 2013

Keywords: parental effects; transcriptome; microarray; hybrid; Salvelinus fontinalis

\section{INTRODUCTION}

In most organisms, the phenotype of an individual is influenced not only by its own genotype and environmental constraints, but also by the genotype and environment of its parents, that is, parental effects. These effects may be defined as the causal mechanisms responsible for the parental influence on the offspring's phenotype (Wolf and Wade, 2009). One simple way to detect parental effects is to produce reciprocal crosses between different strains that are kept in a controlled environment. Thus, if the offspring resulting from the cross between a male of strain A and a female of strain B have a different phenotype from those resulting from the reciprocal cross, an effect of parental strain and sex may be inferred (Roff, 1996; Mousseau and Fox, 1998; Roff and Sokolovska, 2004; Saavedra and Amat, 2005).

Multiple genetic and environmental phenomena have a role in parental effects, including: mate choice, maternal or paternal care, parental allocation of resources, cytoplasmic components inherited from the egg and sperm, and genomic imprinting, in which one parental allele is epigenetically modified. These mechanisms have been reported to influence many traits in several animal and plant species and may have ecological and evolutionary implications by affecting offspring fitness (Mousseau and Fox, 1998; Badyaev and Uller, 2009;
Donelson et al., 2009; Inchausti and Ginzburg, 2009; Wolf and Wade, 2009). Parental effects are also exploited in both aquaculture and agronomy to improve the performance of production stocks in terms of growth and disease resistance, among other traits (Green, 2008).

In fishes, parental effects are particularly known to have an important role in the development at the embryo and juvenile stages (Green, 2008; Donelson et al., 2009) and have been widely documented in salmonid species (Refstie and Steine, 1978; Einum and Fleming, 1999, 2000; Heath et al., 1999; Perry et al., 2004, 2005; Johnson et al., 2007; Nichols et al., 2007; Petersson and Järvi, 2007; Wedekind et al., 2008; Eilertsen et al., 2009). These studies have documented the effects of maternal or paternal phenotypic traits (such as color, egg size, weight and length) on the length, fitness, development rate or growth of offspring. For example, the size of the mother is correlated with the size of the eggs, which in turn influences the length of offspring (Einum and Fleming, 1999; Heath et al., 1999; Green, 2008). Moreover, length and color of the father may be significantly associated with the offspring length (Refstie and Steine, 1978; Perry et al., 2004; Eilersten et al., 2009). In brook charr (Salvelinus fontinalis) and chinook salmon (Oncorhynchus tshawytscha), it has been shown that maternal effects related to growth

${ }^{1}$ Département de Biologie, Institut de Biologie Intégrative et des Systèmes (IBIS), Université Laval, Québec, Québec, Canada and ${ }^{2}$ ISMER, Université du Québec à Rimouski, Rimouski, Québec, Canada

Correspondence: Dr B Bougas, Département de Biologie, Institut de Biologie Intégrative et des Systèmes (IBIS), Pavillon Charles-Eugène Marchand, Université Laval, Québec, Québec, Canada G1V OA6.

E-mail: berenice.bougas.1@ulaval.ca

Received 29 May 2012; revised 3 October 2012; accepted 15 November 2012; published online 9 January 2013 
completely disappear after the yolk sac resorption stage (Heath et al., 1999; Perry et al., 2005; Johnson et al., 2007).

In comparison with the wealth of studies focusing on parental effects on external phenotypes, very few have tried to investigate the impacts of parental effects on the transcriptome. So far, such effects have been investigated using microarrays in only a few Drosophila studies. In particular, Gibson et al. (2004) showed that $2 \%$ of the 12559 analyzed genes in F1 heterozygotes presented parental effects (that is, a resemblance to either paternal or maternal expression). In fishes, previous studies mostly emphasized differences in gene expression during embryogenesis to detect maternal-effect genes across developmental transitions or to detect differences in development rate in embryos (Mathavan et al., 2005; Bai et al., 2007; Nolte et al., 2009; Xu et al., 2010; Renaut et al., 2011). To our knowledge, however, no study has yet systematically focused on investigating the dynamics of parental effects on the transcriptome across different development stages.

In this study, we investigate parental effects on genome-wide transcription profiles, using microarrays, at three different stages of development in a vertebrate species, the brook charr (Salvelinus fontinalis). Brook charr is a salmonid native to eastern North America, where it is highly prized by anglers. It is also an economically important farmed fish in Quebec, Canada, where it represents $57 \%$ of the freshwater aquaculture production in weight (MAPAQ, 2010). F1 crosses descending from three genetically distinct populations, two wild and one domestic, were performed to investigate the phenotypic response relative to parental populations to obtain individuals with improved performances for the aquaculture industry, mostly in terms of enhanced growth and delayed age at sexual maturity. F1 reciprocal crosses were used to detect the parental effects on transcriptome at three stages: hatching, yolk sac resorption and 15 weeks after exogenous feeding. To document specific maternal and paternal effects, parent lengths were measured, as well as offspring lengths at the three stages of development. Our main objective was to test the hypothesis that parental effects acted at the whole transcriptome scale in offspring by modulating gene expression differently among reciprocal offspring. We also tested whether these effects decrease during development, as has been observed for certain phenotypic traits in brook charr (Perry et al., 2005).

\section{MATERIALS AND METHODS}

\section{Fish crosses}

Individual brook charr was produced using breeders from three genetically distinct populations: a Domestic population (D), used by the aquaculture industry in Quebec, Canada, and the Laval (L) and Rupert (R) populations. The $\mathrm{L}$ population is derived from an anadromous population originating from the Laval River near Forestville (north of the St Lawrence River, Quebec), whereas the $\mathrm{R}$ population originates from a freshwater wild population of the Rupert River, which drains into James Bay in north-western Quebec. Breeders from the $\mathrm{L}$ and $\mathrm{R}$ populations were kept in captivity for three generations at the Institut des sciences de la mer de Rimouski (ISMER, Quebec) and at the Laboratoire de Recherche des Sciences Aquatiques (LARSA, Laval University, Quebec), respectively. Breeders from the D population derived from the Nashua and Baldwin stocks were obtained from Pisciculture de la Jacques Cartier (Cap-Santé, Quebec). The three populations are highly genetically differentiated, with a mean $\mathrm{F} s t=0.427 \pm 0.020$ among the $\mathrm{L}$ and $\mathrm{R}$ populations and a mean $\mathrm{F} s t=0.187 \pm 0.009$ among the $\mathrm{D}$ vs the $\mathrm{L}$ and $\mathrm{R}$ populations (Martin et al., 1997). They also differ significantly in patterns of gene transcription when reared in common environment. Thus, the more genetically distant of these populations, $\mathrm{R}$ and $\mathrm{L}$, differently expressed only 72 transcripts, compared with 178 and 191 transcripts with the genetically intermediate (in terms of genetic distance) D population, respectively
(Bougas et al., 2010). In 2005, breeders from the Domestic (D), Laval (L) and Rupert (R) populations were crossed to generate 10 full-sib outbred families per parental cross and per hybrid cross. Two hybrids and reciprocal

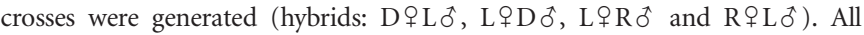
families were kept separately under identical controlled conditions at the Laboratoire de recherche des sciences aquatiques (LARSA, Laval University, Quebec). Fertilized eggs were incubated at $6{ }^{\circ} \mathrm{C}$ up to hatching. The progeny were then kept at $8^{\circ} \mathrm{C}$, under a photoperiod of $12 \mathrm{~h}$ of light and $12 \mathrm{~h}$ of darkness.

\section{Microarray experiments}

Biochips. The transcription profiles of the four crosses were measured using salmonid cDNA microarrays spotted with 16006 features produced by the consortium for Genomic Research on All Salmon Project (cGRASP, available from Ben F. Koop, http://web.uvic.ca/grasp/). The 16006 features come from 175 cDNA libraries constructed from a wide variety of tissues sampled at different stages of development (detailed in von Schalburg et al. (2005)). This chip has been successfully tested and applied to brook charr (Koop et al., 2008; Mavarez et al., 2009; Bougas et al., 2010; Sauvage et al., 2010).

Sampling. Eight families of individual brook charr were sampled for the

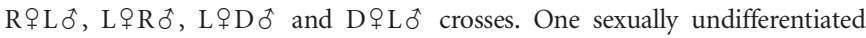
individual per family was randomly sampled at hatching, yolk sac resorption stage, corresponding to the moment where the yolk sac is totally absorbed, and 15 weeks after exogenous feeding. These were immediately frozen in a mix of dry ice and alcohol, for a total of 96 individuals.

RNA extraction, labeling and cDNA hybridization. Total RNA from the 96 deep-frozen individuals was extracted in toto. To remove the effect of egg content, the yolk sacs were removed for all juveniles sampled at the hatching stage. Total RNA was extracted with the PureLink Micro-to-Midi Total RNA Purification System Kit and then treated using DNase I, Amplification Grade (1 unit per $\mu$ l; Invitrogen, Carlsbad, CA, USA) following the manufacturer's protocol. Total RNA was stored in pure water spiked with $1 \mu \mathrm{l}$ Superase-In RNase Inhibitor (Ambion, Foster City, CA, USA) at $-80^{\circ} \mathrm{C}$. Quality and integrity of the total RNA were controlled using an Experion Automated Electrophoresis Station and RNA HighSens Chips (Bio-Rad, Hercules, CA, USA). For each sample, $12 \mu \mathrm{g}$ total RNA was retro-transcribed and the cDNA samples labeled using Genisphere 3DNA Array 50 Kit, Invitrogen's Superscript II retro-transcriptase and Cyanine3 and Alexa 647 fluorescent dyes (Genisphere), following the procedures described at http://web.uvic.ca/cbr/grasp/ (Genisphere Array 50 Protocol). For each developmental stage, cDNA of eight individuals from each cross (corresponding to one individual per family) was hybridized to microarrays, for a total of 48 microarrays. The distribution of dyes was equilibrated among each cross (four samples with Cyanine 3 and four samples with Alexa647 for each developmental stage). Pair-wise direct

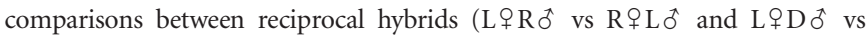
$\mathrm{D} \uparrow \mathrm{L} \delta$ ) were performed at each developmental stage (Churchill, 2002).

Data acquisition, preparation, statistical analysis. Microarrays were scanned using a ScanArray scanner (Perkin-Elmer Life Sciences, Waltham, MA, USA). Spots were localized and quantified with the QuantArray 3.0 software (PerkinElmer Life Sciences), using the histogram quantification method and keeping the mean value of intensity for each spot. Local background was removed and the data from bad spots were manually excluded from the data set. For each of the two dyes on each array, spots with signal intensities lower than the mean intensity of the empty spots plus twice their standard deviation were flagged as non-expressed. Spots that had no non-expressed flag in at least one group (RL, LR, LD or DL) for at least one developmental stage were kept. This left a total of 3411 spots for the analysis which were common to $\mathrm{R}+\mathrm{L} \delta / \mathrm{L}+\mathrm{R} \delta^{-}$and L $9 \mathrm{D} \delta \hat{~} / \mathrm{D}+\mathrm{L} \delta \hat{~}$ comparisons. After a base two logarithm transformation, the data were normalized according to the rlowess method (regional lowess procedure) implemented in R/MAANOVA package (Kerr et al., 2000) to remove signal intensity-dependent and region-dependent dye effects on each slide.

To detect differences in transcription profiles between the crosses at specific stages, data were analyzed using a mixed model ANOVA (Wolfinger et al., 
2001) and the R/MAANOVA package (Kerr et al., 2000, 2002). We tested the presence of cross-type effects with the following ANOVA model:

$$
Y_{i j l}=\mu+G+A_{i}+D_{j}+C_{l(i j)}+\left(A D_{i j}\right)+\left(G A_{i}\right)+\left(G D_{j}\right)+\left(G C_{l(i j)}\right)+\varepsilon_{i j l}
$$

where A: array; $D$ : Dye; $C$ : Cross type; $G$ : Gene; terms in parentheses are interaction terms. This model included 'Array' as a random term and 'Dye' and 'Cross type' as fixed terms. A permutation-based $F$ test (Fs, with 1000 sample ID permutations) was then performed and restricted maximum likelihood was used to solve the mixed model equations (Cui et al., 2005). We tested the presence of cross type effects with the ANOVA model and used the $P$-values to determine the significance of inter-cross differential expression. A False Discovery Rate correction (FDR $=0.05$ ) was applied within the R/MAANOVA package to account for multiple testing.

Gene ontology. Functional classification and assessment of significant differential representation of functional classes were performed in the DAVID (http://david.abcc.ncifcrf.gov/) and PANTHER (http://www.pantherdb.org/) environments. DAVID 2.1 (beta version) gene accession conversion tool (Huang et al., 2009a, b) was used to convert Gene Ontology-linked identifications and Swissprot identifiers from the salmonid microarrays to UNIGENE clusters. Then, the assessment of significant differential representation of functional categories was performed with the 3411 common transcripts serving as the background list in PANTHER (binomial test, significance threshold: $P$-value $=0.05$ ).

\section{Body size measurement and estimation of parental effects}

Sampling. All dams and sires were measured at the time of mating. Twenty sexually undifferentiated offspring per family (ten families for L $+\mathrm{D} \delta \hat{\sigma}, \mathrm{R}+\mathrm{L} \delta$ and $\mathrm{L} \uparrow \mathrm{R} \hat{o}$ crosses, and eight families for $\mathrm{D} \uparrow \mathrm{L} \hat{O}$ cross) were sampled and their fork length measured at hatching and yolk sac resorption stages. For the last sampling stage, 15 weeks after exogenous feeding, 50 individuals per family were sampled and measured.

Data analysis. Normality and homoscedasticity of length data were tested respectively with the Kolmogorov-Smirnov and the Brown and Forsythe tests (Quinn and Keough, 2005). To detect potential cross effects, data were analyzed with the following ANOVA mixed model, implemented in ASReml version 2 (VSN International, Hemel Hempstead, UK):

$$
Y_{i j k}=\mu+C_{i}+F_{j(i)}+\varepsilon_{i j k}
$$

with $C$ (cross-type) as the fixed effect and $F$ (family) nested within $C$ as the random effect. The test was followed by an a posteriori analysis when significant. Differences between means were tested with Tukey tests and, in the case of non-homoscedastic data, the Games and Howell tests, using SPSS version 13.0 (SPSS Inc., Chicago, IL, USA). Length data of breeders and offspring are presented in Supplementary File S1. To estimate the influence of maternal vs paternal length on the length of their offspring, the offspring length at each stage of development was regressed against the length of each of their parents (Falconer and Mackay, 1996). Because parents and offspring were not measured at the same age, and to make the regression slopes comparable among the developmental stages, the length data were standardized to a mean of zero and variance of one by subtracting the sample mean from each sample and dividing this difference by the sample variance. This correction was applied separately to the offspring for each stage and to the dam and sire lengths.

\section{RESULTS}

\section{Parental effects on gene expression}

Significant parental effects were detected and these differed between cross types and development stages within cross type. Thus, only one transcript was significantly differently expressed at hatching. Namely, elongation factor 2 was significantly underexpressed in $\mathrm{L}+\mathrm{R} \delta$ relative to $\mathrm{R}+\mathrm{L} \hat{0}$, whereas no significant difference was observed between

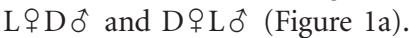

In sharp contrast, many transcripts were differentially expressed at the yolk sac resorption stage and their numbers varied considerably a

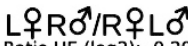
Ratio UE $(\log 2):-0.29$

(1)

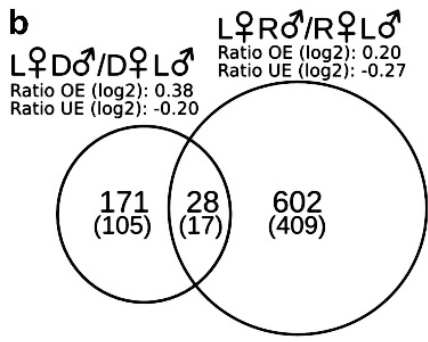

C

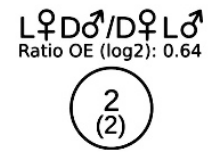

Figure 1 Venn diagrams representing the number of differentially expressed transcripts $(F D R=0.05)$ and the corresponding expression ratio (log2) between the reciprocal crosses ( $\mathrm{L}+\mathrm{D} \delta / \mathrm{D}+\mathrm{L} \delta$ and $\mathrm{L} q \mathrm{R} \delta / \mathrm{R} q \mathrm{~L} \delta$ ) at hatching (a), yolk sac resorption stage (b) and 15 weeks after exogenous feeding (c). Intersection between the circles corresponds to the number of transcripts that are common in the two comparisons. The number of genes represented by the transcripts is indicated in parentheses. The number of genes in parentheses and the corresponding mean of ratio are given. LqDô, Laval female $\times$ Domestic male hybrids; DơLô, Domestic female $\times$ Laval male hybrids; $L$ 우 $\hat{o}$, Laval female $\times$ Rupert male hybrids; $\mathrm{R} \nmid \mathrm{L} \hat{\delta}$, Rupert female $\times$ Laval male hybrids; UE, underexpressed transcripts; $\mathrm{OE}$, overexpressed transcripts.

between the two reciprocal comparisons. Thus, between the L $q \mathrm{D} \delta$ and $\mathrm{D} q \mathrm{~L} \hat{\sigma}$ crosses, 199 transcripts, representing 102 unique genes (genes sharing the same name) and 20 'unknown' transcripts, were differentially expressed, while 630 transcripts, representing 346 unique genes and 80 'unknown' transcripts, were differentially expressed

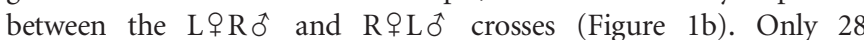
transcripts representing 17 genes were common to the two comparisons (Table 1).

In the $\mathrm{L}+\mathrm{D} \delta / \mathrm{D}+\mathrm{L} \delta$ comparison, the number of transcripts showing overexpression was higher than the number of transcripts showing underexpression with 115 overexpressed transcripts (average $\log _{2}$ of fold change: 0.38 ) and 63 underexpressed transcripts (average $\log _{2}$ of fold change: -0.20 ) for $\mathrm{L}+\mathrm{D} \hat{\sigma}$ relative to $\mathrm{D} q \mathrm{~L} \hat{\delta}$. In the $\mathrm{L}+\mathrm{R} \hat{\sigma} / \mathrm{R}+\mathrm{L} \hat{o}$ comparison, there were 310 overexpressed transcripts (average $\log _{2}$ of fold change: 0.20 ) and 239 underexpressed transcripts (average $\log _{2}$ of fold change: -0.27 ) in $\mathrm{L}+\mathrm{R} \hat{\sigma}$ relative to $\mathrm{R}+\mathrm{L} \hat{0}$. Details regarding all differently expressed genes between the $\mathrm{L} q \mathrm{R} \delta$ and $\mathrm{R}+\mathrm{L} \hat{\sigma}$ crosses, as well as between the $\mathrm{L}+\mathrm{D} \hat{\sigma}$ and $\mathrm{D} \hat{+} \mathrm{L} \hat{\sigma}$ crosses, are given in Supplementary Tables S1 and S2.

At the third sampling period, 15 weeks after exogenous feeding, for the $\mathrm{L}+\mathrm{D} \delta / \mathrm{D} q \mathrm{~L} \delta$ comparison, only two transcripts (genes coding for the ribonucleoprotein A1 and the keratin type I cytoskeletal 13) were

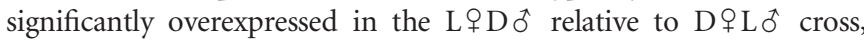
while no transcript was differentially expressed for the $\mathrm{L}+\mathrm{R} \delta \hat{\sigma} / \mathrm{R}+\mathrm{L} \delta \hat{~}$ comparison (Figure 1c).

\section{Gene ontology}

UNIGENE annotation was available for $53.3 \%$ of the cDNA clones that were differently expressed in either or both of the comparisons. 


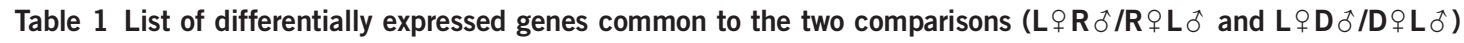

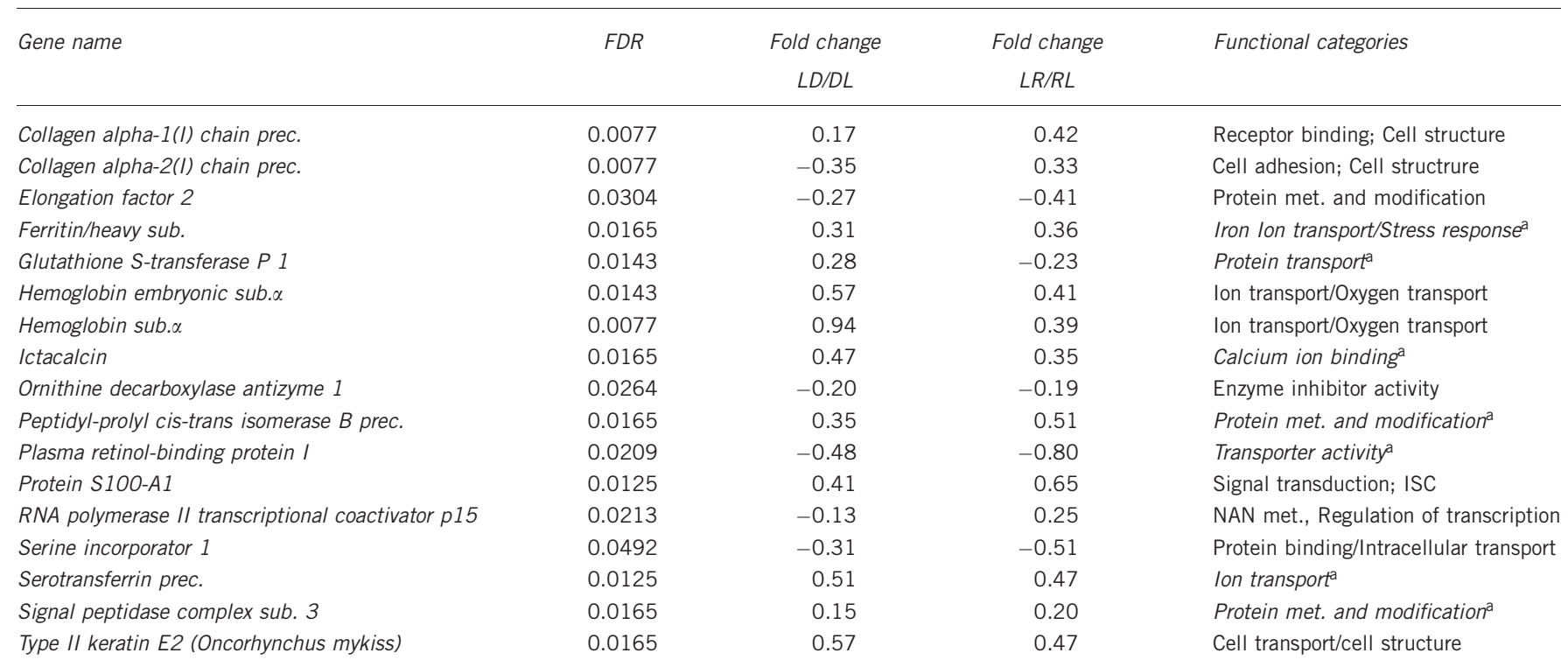

Abbreviations: D, domestic; FDR, false discovery rate; ISC, intracellular signaling cascade; L, laval; Met., metabolism; NAN, nucleotid and acid nucleic; R, rupert.

Fold changes represent the average of base 2 logarithms of the absolute fold changes. FDR corresponds to the FDR-corrected (1000 permutations) P-value of the ANOVA test.

aFunctional categories presented in italic correspond to gene ontology functions for the genes.

All further Gene Ontology analysis was applied only to transcripts that were differentially expressed at the yolk sac resorption stage, which represented all but three of the genes. Only the genes that were automatically annotated were used for binomial test in PANTHER. In addition to these automatically annotated genes, Supplementary Tables S1 and S2 also present genes that were manually annotated by querying the swissprot database. No functional categories were overrepresented among the 17 genes that were common in both

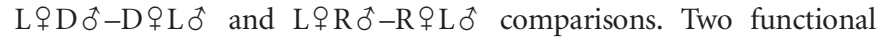
categories were significantly overrepresented among the genes that

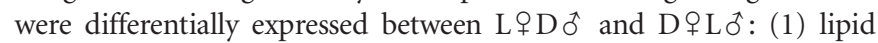
metabolism and (2) signal transduction. These categories were represented by genes that are both underexpressed and overexpressed in the $\mathrm{L}+\mathrm{D} \hat{\sigma}^{\hat{\alpha}}$ cross relative to $\mathrm{D}+\mathrm{L} \hat{0}$ cross (Table 2; Supplementary Table S1). For the $\mathrm{L}+\mathrm{R} \hat{\delta} / \mathrm{R}+\mathrm{L} \hat{\jmath}$ comparison, eight functional categories were overrepresented, which did not include the two that were overrepresented in the $\mathrm{L}+\mathrm{D} \delta \hat{\delta} / \mathrm{D} q \mathrm{~L} \hat{\sigma}$ comparison: cell motility, cell cycle, cell structure, intracellular transport, intracellular protein traffic, muscle contraction and nucleic acid metabolism. The majority of the genes in these categories were overexpressed in the L $q \mathrm{R} \hat{0}$ progeny, including $100 \%$ in cell motility and cell structure, $87 \%$ in cell cycle, $92.3 \%$ in intracellular protein traffic and $89.3 \%$ in nucleic acid metabolism (Table 2; Supplementary Table S2).

\section{Body size differences among crosses and parental effects on} offspring size

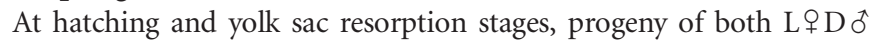
and $\mathrm{L}+\mathrm{R} \hat{\sigma}$ crosses was significantly longer than individuals from their reciprocal crosses. After 15 weeks of exogenous feeding, L + D 0 individuals were still significantly longer than those from the reciprocal cross, whereas the length differences between $\mathrm{L}+\mathrm{R} \delta$ and $\mathrm{R}$ 응 $\widehat{o}$ individuals were no longer significant (Figure 2). The effects of the maternal and paternal lengths on offspring length also differed among crosses and across developmental stages (Figure 3). For the
Table 2 Overrepresented functional categories in the $\mathbf{L} q \mathbf{R} \delta / \mathbf{R}+\mathbf{L} \delta^{\star}$ and $\mathbf{L} \uparrow \mathbf{D} \hat{\delta} / \mathbf{D} q \mathbf{L} \hat{\text { ô }}$ comparisons (binomial test, significance threshold: $P$-value $=0.05$; http://www.pantherdb.org)

\begin{tabular}{|c|c|c|c|c|c|}
\hline Comparison & $\begin{array}{l}\text { Functional } \\
\text { category }\end{array}$ & $\begin{array}{l}\text { Number of } \\
\text { UNIGENE }\end{array}$ & $\% O G$ & $\begin{array}{l}\text { OG fold } \\
\text { change }\end{array}$ & $\begin{array}{l}\text { UG fold } \\
\text { change }\end{array}$ \\
\hline L웋 vs Rótô & Cell cycle & 8 & 87.0 & 0.30 & -0.30 \\
\hline \multirow{6}{*}{$\begin{array}{l}\text { (346 significant } \\
\text { unique genes) }\end{array}$} & Cell motility & 7 & 100.0 & 0.30 & - \\
\hline & Cell structure & 9 & 100.0 & 0.31 & - \\
\hline & $\begin{array}{l}\text { Intracellular } \\
\text { protein traffic }\end{array}$ & 13 & 92.3 & 0.34 & -0.49 \\
\hline & $\begin{array}{l}\text { Intracellular } \\
\text { transport }\end{array}$ & 13 & 61.5 & 0.35 & -0.47 \\
\hline & $\begin{array}{l}\text { Nucleic acid } \\
\text { metabolism }\end{array}$ & 28 & 89.3 & 0.30 & -0.30 \\
\hline & $\begin{array}{l}\text { Muscle } \\
\text { contraction }\end{array}$ & 5 & 40.0 & 0.25 & -0.25 \\
\hline LiDơ vs Dot Lơ & $\begin{array}{l}\text { Lipid } \\
\text { metabolism }\end{array}$ & 5 & 40.0 & 0.48 & -0.36 \\
\hline $\begin{array}{l}\text { (102 significant } \\
\text { unique genes) }\end{array}$ & $\begin{array}{l}\text { Signal } \\
\text { transduction }\end{array}$ & 7 & 28.6 & 0.24 & -0.32 \\
\hline
\end{tabular}

Abbreviations: D, domestic; L, laval; OG, overexpressed gene; UG, underexpressed gene; R, rupert.

Fold changes are log transformed.

L $9 \mathrm{D} \hat{\circ}$ and $\mathrm{D} q \mathrm{~L} \hat{o}$ individuals, the positive maternal effects disappeared after the yolk sac resorption (Figures $3 \mathrm{a}$ and $\mathrm{b}$ ). The length of L $+\mathrm{D} \widehat{o}^{t}$ individuals was positively associated with that of their mother at both the hatching and yolk sac resorption stages. Also, the relative maternal effect was more important at the yolk sac resorption stage compared with the hatching stage. In contrary, L $q \mathrm{R} \hat{\sigma}^{\hat{\sigma}}$ and $\mathrm{R}+\mathrm{L} \delta$ phenotypes showed a negative correlation with their parents' length (Figures $3 \mathrm{c}$ and $\mathrm{d}$ ). 

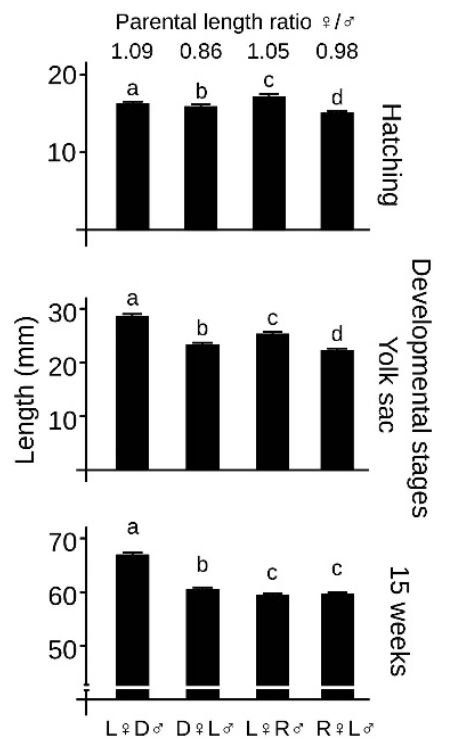

Figure 2 Length of individual brook charr issued from hybrid crosses at three different developmental stages: hatching, yolk sac resorption stage and 15 weeks after exogenous feeding. Mean \pm s.e. Within each development stage, different letters above the bars indicate significantly different means $(P$-value $<0.05)$. Numbers shown above the bar plots correspond to the ratios of female and male lengths for each cross.

\section{DISCUSSION}

\section{Parental influence at different stages of development}

Maternal effects are mediated by egg components and, later, by yolk sac components such as nutriments, hormones, components of defense mechanisms, maternally contributed mitochondria and maternal mRNAs (Kanaya et al., 1996; Løvoll et al., 2006; Bai et al., 2007; Green, 2008). The maternal mRNA present in the egg influences its quality and, consequently, embryonic development. RNA and proteins present in the sperm also contribute to zygote formation and embryo development, although to a lesser extent (Loppin et al., 2005). It has already been suggested that the lower divergence observed in early developmental stages could be explained by the particular constraints placed on the expression of genes responsible for early development. Indeed, these genes are the ones responsible for the phylogenetic organization among eukaryotes and their expression must be tightly controlled in order to lead to the development (Domazet-Lošo and Tautz, 2010). The striking gene expression differences that we observed between the reciprocal hybrids only at the yolk sac resorption stage could be the result of the interaction of yolk components with the offspring genome. For example, yolk sac components, like hormones, proteins or nutriments, which are influenced by the genetic background of the parents, could directly or indirectly influence the offspring's genomic activity by modifying or interacting with either transcription factors or DNA structure. Moreover, Bai et al. (2007) suggested that a high abundance of maternal ribosomes is found in the yolk sac and could have an impact on the translation control of the offspring genome. In addition to these yolk sac components, maternally deposited RNAs are known to degrade relatively slowly and consequently, have been found in embryos up to late developmental stages (Mathavan et al., 2005; Baroux et al., 2008). As a result, these maternal RNAs could act as transcription regulators and interact with the zygote's transcripts to regulate the level of expression of some genes. All of these interactions contribute to the presence of gene expression differences at this stage between reciprocal hybrids, but their complexity limits the possibility of separating the parental from the zygotic contributions. The lack of gene expression differences in reciprocal hybrids 15 weeks after exogenous feeding confirms that the pronounced differences between reciprocal crosses at the yolk sac resorption stage are caused by parental deposits, which disappear at later stages. Another possible explanation could be that the transcriptional differences are driven by specific tissues or cells that are present in a smaller proportion in whole individuals at this specific stage of development relative to whole individuals at the yolk resorption stage. Such a small proportion could have prevented detecting the transcriptional differences at the level of the whole individuals. To verify this hypothesis, it would relevant that future studies aim at measuring the transcriptional differences in specific cell or tissues among juvenile brook charr.

\section{Parental influence among cross-types}

Our results also revealed pronounced differences in gene expression across the two groups of reciprocal crosses. In fact, many more transcripts were differently expressed between the $\mathrm{L} q \mathrm{R} \hat{\sigma} / \mathrm{R}+\mathrm{L} \hat{0}$

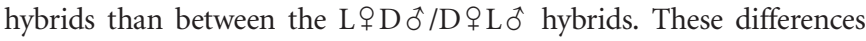
could be caused by intrinsic differences in the genetic architecture of the parents which consequently would interact differently when crossed together. These differences could in turn be exacerbated when parental yolk sac deposits interact with the offspring zygote. In support of this hypothesis, results from our previous study comparing hybrids with parental populations at the yolk sac resorption stage showed that hybrids from the $\mathrm{L}$ and $\mathrm{R}$ crosses and the $\mathrm{L}$ and $\mathrm{D}$ crosses had unpredictable transcriptomic responses (Bougas et al., 2010). Relative to their parental populations, these two hybrid crosses presented differences in terms of which genes were differentially expressed, as well as in the major modes of transcription regulation (that is, additivity, dominance and over- or underdominance). Dominant or over-underdominant interaction of hybrid genomes is likely responsible for the unpredictable nature of the transcriptomic responses. Moreover, $\mathrm{L} q \mathrm{D} \delta$ individuals displayed $85 \%$ of dominance in gene expression regulation, whereas the $\mathrm{R}+\mathrm{L} \hat{0}$ individuals displayed only $26 \%$ of gene expression dominance (Bougas et al., 2010). This is in accordance with the higher gene transcription misregulation observed in hybrids of the two more divergent populations, $\mathrm{R}$ and $\mathrm{L}$. This transcription misregulation could in turn explain why the reciprocal $\mathrm{R}+\mathrm{L} \hat{\sigma}$ and $\mathrm{L}+\mathrm{R} \hat{\sigma}$ individuals are less similar to one another than the reciprocal $\mathrm{L}+\mathrm{D} \hat{\sigma}$ and $\mathrm{D}+\mathrm{L} \delta$ individuals.

Gene expression differences between the reciprocal crosses could also have influenced the observed differences in length and developmental timing. For example, functional categories such as cell cycle, cell motility, cell structure, intracellular protein traffic and nucleic acid metabolism, that showed a majority of overexpressed genes in $\mathrm{L} q \mathrm{R} \hat{o}$ compared with $\mathrm{R} q \mathrm{~L} \hat{o}$, are consistent with gene expression differences observed during embryonic development studies in other salmonid species, namely lake whitefish (Coregonus clupeaformis) and rainbow trout (Oncorhynchus mykiss). For example, genes involved in the cell cycle and nucleotide and nucleic acid metabolism functional categories were found to be differentially expressed between two ecotypes of lake whitefish at the embryonic stage, suggesting their importance in transcription regulation and development of the embryos (Nolte et al., 2009). In addition, genes involved in these two functional categories were also found to be overexpressed in fast developing rainbow trout individuals ( $\mathrm{Xu}$ et al., 2010). Thus, the higher length of $\mathrm{L} q \mathrm{R} \delta$ relative to $\mathrm{R}+\mathrm{L} \hat{\delta}$ hybrids at the yolk sac resorption stage, and faster development rate from hatching to yolk 


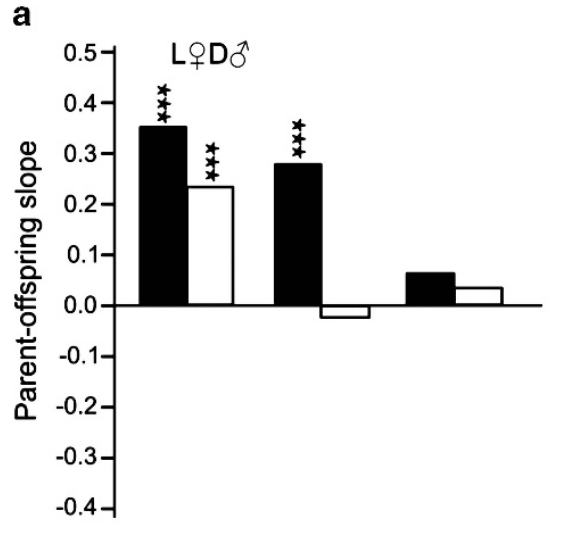

C

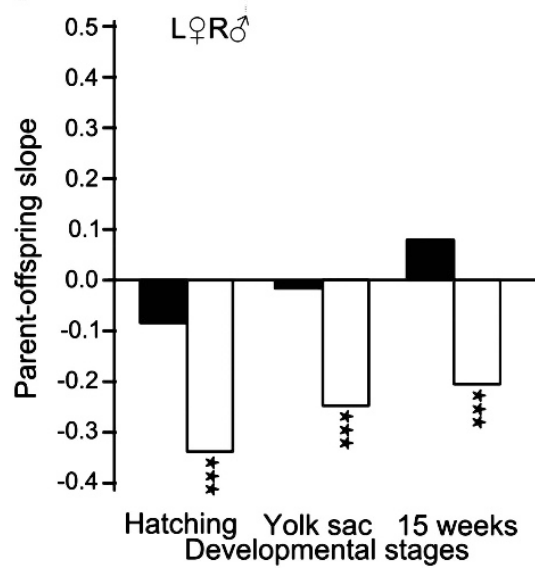

b

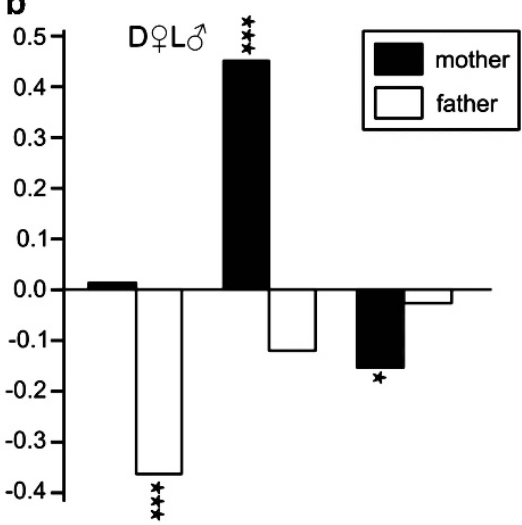

d

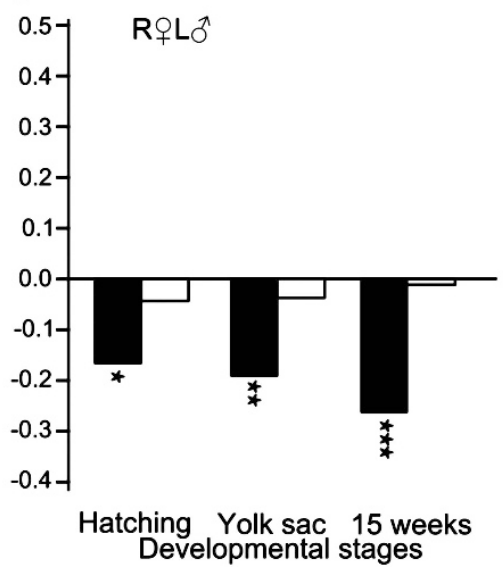

Figure 3 Bar chart showing the regression slopes of offspring length on parent length. Dam-offspring (dark bars) and sire-offspring (light bars) regression slopes are shown at hatching, yolk sac resorption stage and 15 weeks after exogenous feeding. The significant slopes are indicated with the following code: ${ }^{* * *} P$-value $<0.001,{ }^{* *} P$-value $<0.01$ or ${ }^{*} P$-value $<0.05$. A significant positive slope corresponds to positive correlation of parent and offspring lengths, while a significant negative slope corresponds to negative correlation between parent and offspring lengths. (a) Parent-offspring correlation slope for $\mathrm{L}+\mathrm{D} \delta{ }^{*}$

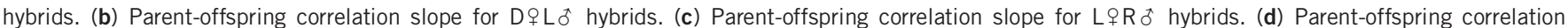
slope for R $\mathrm{L} \mathrm{L}{ }^{\top}$ hybrids.

sac resorption, could be associated with the great number of genes that were differentially expressed at this stage. A possible link between phenotype and overrepresentation of genes related to signal transduction and lipid metabolism is more difficult to interpret because of the nature of the genes involved. They are both less numerous and potentially involved in other functions (Table 2). Namely, the genes related to signal transduction, the mechanism responsible for conversion of the mechanical/chemical stimuli into specific cellular responses, also have a role in cellular reorganization and ligand binding receptors. Among these genes, two overexpressed genes in L옹 relative to $\mathrm{D}$ 잉 (calcium/calmodulin-dependent protein kinase and protein S100) are involved in transcription regulation and cell growth differentiation. Two underexpressed genes are involved in apoptosis and regulation of cell growth (growth arrest and DNA damage-inducible protein GADD45 beta and stathmin). Moreover, the regulation of stathmin protein is controlled by the calcium/calmodulin-dependent protein kinase protein (Cassimeris, 2002). Upregulation of these genes could also have promoted the higher growth in the L잉 individuals (Figure 2). Genes related to lipid metabolism have a role in the processes of synthesis and degradation of lipids. One gene responsible for fatty acid protein transport in cells (fatty acid-binding protein, intestinal gene) was overexpressed in the LqDô individuals, while one gene involved in fatty acid degradation (acyl-coA-binding protein gene) was underexpressed. These processes could have favored the accumulation of fatty acids and thus could be responsible in part for the higher weights observed in these individuals. Upregulation of genes involved in growth, intracellular and extracellular transport, cell proliferation (such as connective tissue growth factor gene, zymogen granule, hemoglobin, and keratin genes) may also contribute to the greater lengths of L $+\mathrm{D} \delta$ individuals. However, future studies should aim to learn more about some of the specific genes suggested in the present study and their specific effect on fish length. These studies could use qPCR, protein assay and detection to determine the effect of those genes specific on length at age variation among juvenile brook charr.

\section{Specific parental effect on offspring length}

Since our microarray experiment was designed to test for the occurrence of parental effects, the respective roles of maternal vs paternal effects acting on the transcriptional differences could not be disentangled. Previous studies in Drosophila that investigated parental effects at the gene transcription level did not clearly reveal a more pronounced influence of the effect of either parent on the offspring transcriptome. Thus, Gibson et al. (2004) showed that genes in reciprocal hybrid crosses were expressed at a similar level to one of the two parents, but not systematically to that of the mother. Wittkopp 
et al. (2006) also found that gene expression differences among reciprocal crosses in Drosophila were affected by both trans-acting maternal and paternal effects, which the authors imputed to the cytoplasmic contributions of the egg and sperm.

Here, we were able to assess specific paternal and maternal contributions to length phenotype of their progeny. Parental effects have previously been reported to act on phenotypic differences observed among salmonid offspring from early developmental stages, soon after fertilization, and up to the later stages of development (review by Green (2008)). More precisely, in brook charr, the length of offspring at hatching time has been shown to be influenced by the mother's length, which in turn influences the egg, yolk sac and embryo lengths (Perry et al., 2004).

In Arctic charr juveniles, the early growth also seems to be partly determined by paternal effects (Eilersten et al., 2009). Here, our results on length revealed a significant correlation between offspring and mother length as well as father length, as previously found by Perry et al. (2004) for brook charr, and in concordance with the study of Eilersten et al. (2009). Thus, all crosses produced by L females that were longer than their male mate (parental length ratio $>1$ ) were significantly longer than their reciprocal crosses (Figure 2). Maternal effects on length phenotype that were only observed as a significant positive length correlation in the reciprocal $\mathrm{L} q \mathrm{D} \delta$ and $\mathrm{D} q \mathrm{~L} \hat{\sigma}$ hybrids in earlier developmental stages decreased near zero 15 weeks after exogenous feeding (Figure 3a). The decreasing influence of maternal effects is also consistent with previous studies that showed a reduction of the parental effects from hatching onwards and no influence of these effects on later life-history traits (Heath and Blouw, 1998; Lindholm et al., 2006). However, significant negative effect of $\mathrm{R}$ parental population length persisted in the reciprocal $\mathrm{L}+\mathrm{R} \hat{\sigma}$ and $\mathrm{R}+\mathrm{L} \hat{\sigma}$ hybrids through the three stages of development (Figures $3 \mathrm{c}$ and $\mathrm{d}$ ). This negative effect could hypothetically arise from differences in egg quality since female size may influence egg size but not necessarily quality. Egg quality could be influenced by the genetic makeup of the parents (reviewed by Brooks et al. (1997)). Indeed, parental populations used in this study presented marked differences in gene expression, genetic distance and phenotype (Bougas et al., 2010). The different lengths observed among reciprocal hybrids, sharing a similar genetic background, could thus lie in different mobilization or quality of parental factors. However, length is a quantitative trait known to be affected by numerous genes, each having a small effect ( $\mathrm{Li}$ et al., 2009). This makes it more difficult to interpret the specific influence of maternal and paternal effects on the length phenotype.

In conclusion, our results provide evidence for parental effects on gene transcription regulation in brook charr reciprocal crosses and demonstrate that parental effects can contribute to the differences observed between hybrid crosses by modulating the transcriptome in the zygotes. From these results, it is clear that parental effects are complex and vary both in time and depending on the phenotype being observed. We also show that they are highly dependent on the genetic architecture of the parents. The fact that no transcription effects were observed at hatching while parental effects on length already existed at this stage also highlight the importance of nontranscriptomic deposits, such as the presence of nutriments that could act before the yolk sac resorption stage.

\section{DATA ARCHIVING}

Microarray data have been deposited with the NCBI/Gene Expression Omnibus (GEO) under accession number: GSE42435.
Length measurement data have been deposited at Dryad under doi:10.5061/dryad.m6n75.

\section{CONFLICT OF INTEREST}

The authors declare no conflict of interest.

\section{ACKNOWLEDGEMENTS}

We wish to thank the staff of the LARSA (Laboratoire de Recherche des Sciences Aquatiques), and of the ISMER (Institut des sciences de la mer de Rimouski) station, as well as the members from Bernatchez's and Audet's laboratories for their assistance in rearing and sampling the fish used in this study. We also thank S Granier for measurement and first analysis of length data, and E Normandeau and C Sauvage for rich discussions and assistance with the analyses. We are also grateful to Managing editor Roger Butlin and anonymous referees for the constructive comments and suggestions. This study was supported by a research grant form Science and Engineering Research Canada (NSERC strategic program) to L Bernatchez, C Audet and D Cyr. This work is a contribution to the research program of the RAQ (Réseau Aquaculture Quebec).

Badyaev A, Uller T (2009). Parental effects in ecology and evolution: mechanisms, processes and implications. Philos Trans R Soc Lond B Biol Sci 364: 1169-1177.

Bai J, Solberg C, Fernandes J, Johnston I (2007). Profiling of maternal and developmentalstage specific mRNA transcripts in Atlantic halibut Hippoglossus hippoglossus. Gene 386: 202-210.

Baroux C, Autran D, Gillmor C, Grimanelli D, Grossniklaus U (2008). The maternal to zygotic transition in animals and plants. Cold Spring Harb Symp Quant Biol 73: 89-100.

Bougas B, Granier S, Audet C, Bernatchez L (2010). The transcriptional landscape of cross-specific hybrids and its possible link with growth in brook charr (Salvelinus ontinalis Mitchill). Genetics 186: 97-107.

Brooks S, Tyler CR, Sumpter JP (1997). Egg quality in fish: what makes a good egg? Rev Fish Biol and Fish 7: 387-416

Cassimeris L (2002). The oncoprotein 18/stathmin family of microtubule destabilizers. Curr Opin Cell Biol 14: 18-24.

Churchill G (2002). Fundamentals of experimental design for cDNA microarrays. Nat Genet 32: 490-495.

Cui X, Hwang J, Qiu J, Blades N, Churchill G (2005). Improved statistical tests for differential gene expression by shrinking variance components estimates. Biostatistics 6: 59-75.

Domazet-Lošo T, Tautz D (2010). A phylogenetically based transcriptome age index mirrors ontogenetic divergence patterns. Nature 468: 815-818.

Donelson J, Munday P, McCormick M (2009). Parental effects on offspring life histories: when are they important? Biol Lett 5: 262-265.

Eilertsen E, Bardsen B, Liljedal S, Rudolfsen G, Folstad I (2009). Experimental evidence for paternal effects on offspring growth rate in arctic charr (Salvelinus alpinus). Philos Trans R Soc Lond B Biol Sci 276: 129-136.

Einum S, Fleming I (1999). Maternal effects of egg size in brown trout (Salmo trutta): norms of reaction to environmental quality. Proc $R$ Soc Lond B Biol Sci 266: 2095-2100.

Einum S, Fleming I (2000). Selection against late emergence and small offspring in atlantic salmon (Salmo salar). Evolution 54: 628-639.

Falconer D, Mackay T (1996). Introduction to Quantitative Genetics, 4th edn. Longman Essex.

Gibson G, Riley-Berger R, Harshman L, Kopp A, Vacha S, Nuzhdin S et al. (2004). Extensive sex-specific nonadditivity of gene expression in Drosophila melanogaster. Genetics 167: 1791-1799.

Green B (2008). Maternal effects in fish populations. Adv Mar Biol 54: 1-105.

Heath D, Fox C, Heath J (1999). Maternal effects on offspring size: variation through early development of chinook salmon. Evolution 53: 1605-1611.

Heath DD, Blouw DM (1998). Maternal effects in fish: are they adaptive or merely physiological side-effects? In: Mousseau TA, Fox CW (Eds) Adaptive Maternal Effects. Oxford University Press: Oxford, pp 178-201.

Huang DW, Sherman BT, Lempicki RA (2009a). Systematic and integrative analysis of large gene lists using DAVID Bioinformatics Resources. Nat Protoc 4: 44-57.

Huang DW, Sherman BT, Lempicki RA (2009b). Bioinformatics enrichment tools: paths toward the comprehensive functional analysis of large gene lists. Nucleic Acids Res 37: $1-13$.

Inchausti P, Ginzburg L (2009). Maternal effects mechanism of population cycling: a formidable competitor to the traditional predator-prey view. Philos Trans $R$ Soc Lond $B$ Biol Sci 364: 1117-1124.

Johnson R, Shrimpton J, Cho G, Heath D (2007). Dosage effects on heritability and maternal effects in diploid and triploid chinook salmon (Oncorhynchus tshawytscha). Heredity 98: 303-310. 
Kanaya S, Kudo Y, MokiToda S, Katsura K, DelCarpio C (1996). Synchronous gene expressions during embryogenesis of Oncorhynchus masou (Yamame). Biochem Mol Biol Int 39: 261-266.

Kerr M, Afshari C, Bennett L, Bushel P, Martinez J, Walker NJ et al. (2002). Statistical analysis of a gene expression microarray experiment with replication. Stat Sinica 12 203-217.

Kerr M, Martin M, Churchill G (2000). Analysis of variance for gene expression microarray data. J Comput Biol 7: 819-837.

Koop B, von Schalburg K, Leong J, Walker N, Lieph R, Cooper GA et al. (2008). A salmonid EST genomic study: genes, duplications, phylogeny and microarrays. BMC Genomics 9: 545.

Li N, Das K, Wu R (2009). Functional mapping of human growth trajectories. J Theor Biol 261: 33-42.

Lindholm A, Hunt J, Brooks R (2006). Where do all the maternal effects go? Variation in offspring body size through ontogeny in the live-bearing fish Poecilia parae. Biol Lett 2 : 586-589.

Loppin B, Lepetit D, Dorus S, Couble P, Karr T (2005). Origin and neofunctionalization of a drosophila paternal effect gene essential for zygote viability. Curr Biol 15: 87-93.

Løvoll M, Kilvik T, Boshra H, Bøgwald J, Sunyer J, Dalmo RA (2006). Maternal transfer of complement components c3-1, c3-3, c3-4, c4, c5, c7, bf, and df to offspring in rainbow trout (Oncorhynchus mykiss). Immunogenetics 58: 168-179.

MAPAQ (2010). Les pêches et aquaculture commerciales. Bilan 2008 et perspectives (les). Bibliothèque et archives du Québec (Ed.): Québec, QC, CANADA.

Martin S, Savaria J, Audet C, Bernatchez L (1997). Microsatellites reveal no evidence for inbreeding effects but low inter-stock genetic diversity among brook charr stocks used for production in Quebec. Bull Aquacult Assoc Can 97: 21-23.

Mathavan S, Lee S, Mak A, Miller L, Murthy K, Govindarajan K et al. (2005). Transcriptome analysis of zebrafish embryogenesis using microarrays. PLoS Genet 1: 269-276.

Mavarez J, Audet C, Bernatchez L (2009). Major disruption of gene expression in hybrids between young sympatric anadromous and resident populations of brook charr (Salvelinus fontinalis Mitchill). J Evol Biol 22: 1708-1720.

Mousseau T, Fox C (Eds) (1998). Maternal Effects as Adaptations. Oxford University Press: New York.

Nichols K, Broman K, Sundin K, Young J, Wheeler P, Thorgaard G (2007). Quantitative trait loci $x$ maternal cytoplasmic environment interaction for development rate in Oncorhynchus myhiss. Genetics 175: 335-347.

Nolte A, Renaut S, Bernatchez L (2009). Divergence in gene regulation at young life history stages of whitefish (Coregonus sp.) and the emergence of genomic isolation. BMC Evol Biol 9: 59.
Perry G, Audet C, Bernatchez $L$ (2005). Maternal genetic effects on adaptive divergence between anadromous and resident brook charr during early life history. J Evolution Biol 18: 1348-1361.

Perry G, Audet C, Laplatte B, Bernatchez L (2004). Shifting patterns in genetic control at the embryo-alevin boundary in brook charr. Evolution 58: 2002-2012.

Petersson E, Jarvi T (2007). Characteristics of brown trout males influence growth and survival of their offspring. J Fish Biol 71: 493-509.

Quinn G, Keough M (2005). Experimental Design and Data Analysis for Biologists, 4th edn Cambridge University Press: Cambridge, (UK).

Refstie T, Steine T (1978). Selection experiments with salmon. III. Genetic and environmental sources of variation in length and weight of Atlantic salmon in freshwater phase. Aquaculture 14: 221-234.

Renaut S, Bernatchez L (2011). Transcriptome-wide signature of hybrid breakdown associated with intrinsic reproductive isolation in lake whitefish species pairs (Coregonus spp. salmonidae). Heredity 106: 1003-1011.

Roff D (1996). Evolutionary Quantitative Genetics. Chapman \& Hall(Ed.). New York.

Roff D, Sokolovska N (2004). Extra-nuclear effects on growth and development in the sand cricket Gryllus firmus. J Evol Biol 17: 663-671.

Saavedra C, Amat F (2005). Maternal effects on encystment in crosses between two geographic strains of Artemia franciscana. J Hered 96: 713-717.

Sauvage C, Derome N, Normandeau E, St-Cyr J, Audet C, Bernatchez L (2010). Fast transcriptional responses to domestication in the brook charr Salvelinus fontinalis. Genetics 185: 105-112.

von Schalburg K, Rise M, Cooper G, Brown G, Gibbs A, Nelson C et al. (2005). Fish and chips: various methodologies demonstrate utility of a 16,006-gene salmonid microarray. BMC Genomics 6: 126.

Wedekind C, Jacob A, Evanno G, Nusslé S, Müller R (2008). Viability of brown trout embryos positively linked to melanin-based but negatively to carotenoid-based colours of their fathers. Proc Biol Sci 275: 1737-1744.

Wittkopp P, Haerum B, Clark A (2006). Parent-of-origin effects on mRNA expression in Drosophila melanogaster not caused by genomic imprinting. Genetics 173: 1817-1821.

Wolf J, Wade M (2009). What are maternal effects (and what are they not)? Philos Trans $R$ Soc Lond B Biol Sci 364: 1107-1115.

Wolfinger R, Gibson G, Wolfinger E, Bennett L, Hamadeh H, Bushel P et al. (2001). Assessing gene significance from cDNA microarray expression data via mixed models. J Comput Biol 8: 625-637.

Xu P, McIntyre LM, Scardina J, Wheeler P, Thorgaard G, Nichols K (2010). Transcriptome profiling of embryonic development rate in rainbow trout advanced backcross introgression lines. Mar Biotechnol 13: 215-231.

Supplementary Information accompanies the paper on Heredity website (http://www.nature.com/hdy) 